\title{
EVALUADORES NÚMERO 8
}

Con nuestro agradecimiento a los colegas que han participado de este número:

Dra. Alejandra Boni Aristizabal, INGENIO, Universidad Politécnica de Valencia, España

Dra. Nora Sforza, Facultad de Filosofía y Letras, UBA, Argentina

Dra. Graciela Cruz Jiménez, Universidad Autónoma de México (UNAM)

Dra. Cristina Girardo, El Colegio Mexiquense, México

Dra. Cecilia Cadena Inostroza, El Colegio Mexiquense, México

Dra. Gabriela Martinez Tiburcio, Universidad Autónoma de México (UNAM)

Dra. María Dilia Mieles Barrera, Universidad del Magdalena, Colombia

Dr. Arturo Venancio, Universidad Autónoma de México (UNAM)

Dra Ana María Fernández, CIG-UNICEN, Argentina

Dra. María Elisa Gentile, UNICEN, Argentina

Dra. Natasha Picone, CONICET, Argentina

Mag. Marisa Sagua, IHAM-UNMdP, Argentina

Dra. Irma De Felippis, UNTREF, Argentina

Mag. Norma del Río Lugo, Universidad Autónoma Metropolitana, México

Dra. Lia Rodriguez de la Vega, Universidad de Palermo, Argentina

Dra. Denise Benatuil, Universidad de Palermo, Argentina 